\title{
GLOBAL CLASSICAL SOLUTIONS TO THE CAUCHY PROBLEM FOR A NONLINEAR WAVE EQUATION
}

\author{
by \\ HAROLDO R. CLARK \\ Universidade Federal Fluminense \\ Instituto de Matemática - GAN \\ Rua S. Paulo, 30 \\ 24.040-110 Niterói, RJ - Brasil \\ e-mail: ganhrc@vm.uff.br.
}

(Received August 24, 1995 and in revised form January 3, 1997)

ABSTRACT. In this paper we consider the Cauchy problem

$$
\begin{cases}u^{\prime \prime}+M\left(\left|A^{\frac{1}{2}} u\right|^{2}\right) & A u=0 \quad \text { in } \quad] 0, T[ \\ u(0)=u_{0}, & u^{\prime}(0)=u_{1},\end{cases}
$$

where $u^{\prime}$ is the derivative in the sense of distributions and $\left|A^{\frac{1}{2}} u\right|$ is the $H$-norm of $A^{\frac{1}{2}} u$. We prove the existence and uniqueness of global classical solution.

KEY WORDS AND PHRASES: Nonlinear, wave equation, global classical solution 1995 AMS SUBJECT CLASSIFICATION CODES: 35D10; 35005; 35105.

\section{INTRODUCTION}

In this work we prove the existence and uniqueness of global classical solution to the Cauchy problem

$$
\left\{\begin{array}{l}
\frac{\partial^{2} u}{\partial t^{2}}-M\left(\int_{\Omega}|\nabla u(x, t)|^{2} d x\right) \Delta u=0 \\
u(x, 0)=u_{0}(x), \quad \frac{\partial u}{\partial t}(x, 0)=u_{1}(x)
\end{array}\right.
$$

where $\Omega$ is a bounded or unbounded open set of $\mathbb{R}^{n}, M(\xi)$ is a locally Lipschitz function on $\left[0,+\infty\left[, \Delta=\sum_{z=1}^{n} \frac{\partial^{2}}{\partial x_{2}^{2}}\right.\right.$ is the Laplace operator and $|\nabla u(x, t)|^{2}=\sum_{z=1}^{n}\left|\frac{\partial u}{\partial x_{2}}\right|^{2}$.

The equation $(1.1)_{1}$ to model the small vibrations of an elastic string where we admit only vertical component for the tension (cf. Carrier [2]). 
The Cauchy problem (1.1) can be written in an abstract framework, if the Laplace operator is replaced by a self-adjoint positive operator $A$ in a real Hilbert space $H$. Representing by $A^{\frac{1}{2}}$ the square root of $A$, the problem (1.1) have the following abstract framework

$$
\left\{\begin{array}{l}
\left.u^{\prime \prime}+M\left(\left|A^{\frac{1}{2}} u\right|^{2}\right) A u=0 \quad \text { in } \quad\right] 0, T[ \\
u(0)=u_{0}, \quad u^{\prime}(0)=u_{1},
\end{array}\right.
$$

where $u^{\prime}$ is the derivative in the sense of distributions and $\left|A^{\frac{1}{2}} u\right|$ is the $H$-norm of $A^{\frac{1}{2}} u$.

Several authors have been studing the problem (1.1), among them, we can mention the following ones: Arosio-Spagnolo [1], Dickey [3], Ebihara et.al. [4], Lions [8], Matos [9], Medeiros and Milla Miranda [10], Menzala [11], Nishihara [12], Pohozaev [13], Yamada [14]. In Dickey [3] the problem (1.1) was studied for the case $n=1$, when $\Omega$ is the positive real line. His result was generalized for $\Omega=\mathbb{R}^{n}$ by Menzala [11]. These two results were obtained by the method of Fourier transforms and the results proved by Dickey [3] and Menzala [11] was generalized by Matos [9], as an application of the Diagonalization Theorem by Von Neumann-Dixmier, e.g. Huet [5] and Lions-Magenes [6].

To treat the abstract case, when one does not have compactness, Lions [7]-[8] proposed to study the Cauchy problem (1.2) by making use of the Diagonalization Theorem. Therefore, the main objective of this work is to study the problem (1.2), independent of compactness.

To obtain the global classical solutions we suppose that the initial data are analytic-type. With this choice for $u_{0}$ and $u_{1}$, we follow the ideas of Arosio-Spagnolo [1] that prove the global existence of a solution to the Cauchy problem (1.2) when the domain $D(A)$ of the operator $A$ have compact imersion on the real Hilbert space $H$ and the $M(\xi)$ is a nonnegative locally Lipschitz function.

The arguments developed in the present work study the Cauchy problem (1.2) and can be summarized as follows. We apply formally a diagonalization operator $\mathcal{U}$ in the problem (1.2) to obtain

$$
\left\{\begin{array}{l}
v^{\prime \prime}+M\left(\left|\lambda^{\frac{1}{2}} v\right|_{0}^{2}\right) \lambda v=0 \\
v(0)=v_{0}, \quad v^{9}(0)=v_{1},
\end{array}\right.
$$

where $\lambda$ is a positive real parameter, e.g., Section 2. The solution of the Cauchy problem for (1.3) is obtained as the limit $v$, in an appropriated topology of a sequence $\left(v_{k}\right)_{k \in N}$ where $v_{k}$, for each $k$, is a solution of a "Truncated Problem"; e.g. Section 3. The solution $u$ of (1.2) is given by $u=\mathcal{U}^{-1}(v)$. 


\section{TERMINOLOGY}

A field of the Hilbert spaces is, by definition, is a mapping $\lambda \rightarrow \mathcal{H}(\lambda)$, that for each $\lambda \in \mathbb{R}$ is associated a Hilbert space $\mathcal{H}(\lambda)$. A vector field on real number $\mathbb{R}$ is a mapping $\lambda \rightarrow u(\lambda)$ defined on $\mathbb{R}$, such that $u(\lambda) \in \mathcal{H}(\lambda)$.

We represent by $\mathcal{F}$ the real vector space of all vector fields on $\mathbb{R}$ and by $\nu$ a positive real measure.

A field of Hilbert spaces $\lambda \rightarrow \mathcal{H}(\lambda)$ is said to be $\nu$-measurable when there exists a subspace $\mathcal{M}$ of $\mathcal{F}$ satisfying the following conditions:

- The mapping $\lambda \rightarrow\|u(\lambda)\|_{\mathcal{H}(\lambda)}$ is $\nu$-measurable for all $u \in \mathcal{M}$;

- If $u \in \mathcal{F}$ and $\lambda \rightarrow(u(\lambda), v(\lambda))_{\mathcal{H}(\lambda)}$ is $\nu$-measurable for all $v \in \mathcal{M}$, then $u \in \mathcal{M}$;

- There exists in $\mathcal{M}$ a sequence $\left(u_{n}\right)_{n \in N}$ such that $\left(u_{n}(\lambda)\right)_{n \in N}$ is total on $\mathcal{H}(\lambda)$, for each $\lambda \in \mathbb{R}$.

The objects of $\mathcal{M}$ are called $\nu$-measurable vector fields. In the following, $\lambda \rightarrow \mathcal{H}(\lambda)$ represents a $\nu$-measurable field of Hilbert spaces and all the vector fields considered are $\nu$ measurable.

Next we define the space $\mathcal{H}_{0}=\int{ }^{\oplus} \mathcal{H}(\lambda) d \nu(\lambda)$ in the following way: a vector field $\lambda \rightarrow$ $u(\lambda)$ is in $\mathcal{H}_{0}$ if only if

$$
\int_{\boldsymbol{R}}\|u(\lambda)\|_{\mathcal{H}(\lambda)}^{2} d \nu(\lambda)<+\infty
$$

Two vector fields that are equal a.e., in $\mathcal{H}_{0}$, relative to the measure $\nu$, will be identified. We define in $\mathcal{H}_{0}$ the scalar product:

$$
(u, v)_{0}=\int_{\boldsymbol{R}}(u(\lambda), v(\lambda))_{\mathcal{H}(\lambda)} d \nu(\lambda), \quad \text { for all } u, v \in \mathcal{H}_{0} .
$$

With the scalar product $(2.1)$ the vector space $\mathcal{H}_{0}$ turns out to be a Hilbert space which is called the hilbertian integral (or, as called by other authors, measurable hilbertian sum; e.g. Lions and Magenes [6]) of the field $\lambda \rightarrow \mathcal{H}(\lambda)$.

Given a real number $\alpha$, denote by $\mathcal{H}_{\alpha}$ the Hilbert space of the vector fields $u$ such that the field $\lambda \rightarrow \lambda^{\alpha} u(\lambda)$ is in $\mathcal{H}_{0}$. In $\mathcal{H}_{\alpha}$ we define the following norm

$$
|u|_{\alpha}^{2}=\left|\lambda^{\alpha} u\right|_{0}^{2}=\int_{\boldsymbol{R}} \lambda^{2 \alpha}|| u(\lambda) \|_{\mathcal{H}(\lambda)}^{2} d \nu(\lambda), \quad u \in \mathcal{H}_{\alpha}
$$

Let us fix a separable Hilbert space $H$ with scalar product $($,$) and norm |, |. We consider$ in $H$ a selfadjoint operator $A$ such that

$$
(A u, u) \geq \beta|u|^{2}, \quad \text { for all } u \in D(A), \quad \beta>0 .
$$


where $D(A)$ is the domain of $A$. With this hypothesis, the operator $A$ satisfies the conditions of the Diagonalization Theorem, e.g. [6]. It then follows that there exists a Hilbertian integral $\mathcal{H}_{0}=\int^{\oplus} \mathcal{H}(\lambda) d \nu(\lambda)$, where $\nu$ is a positive Radon measure with support in $] \lambda_{0},+\infty\left[, 0<\lambda_{0}<\right.$ $\beta$ (where $\beta$ is the constant of (2.3), and a unitary operator $\mathcal{U}$ from $H$ onto $\mathcal{H}_{0}$, such that:

$$
\mathcal{U}\left(A^{\alpha} u\right)=\lambda^{\alpha} \mathcal{U}(u), \quad \text { for all } u \in D\left(A^{\alpha}\right), \quad \alpha \geq 0
$$

where $D\left(A^{\alpha}\right)$ is equipped with the graph norm, i.e.,

$$
|u|_{D\left(A^{\alpha}\right)}^{2}=|u|^{2}+\left|A^{\alpha} u\right|^{2}, \quad \text { for all } u \in D\left(A^{\alpha}\right)
$$

Observe that with the $\operatorname{supp}(\nu) \subset] \lambda_{0},+\infty[$, we have for $\alpha \geq \beta, \alpha$ and $\beta$ real number, that

$$
\begin{gathered}
\mathcal{H}_{\alpha} \subset \mathcal{H}_{\beta} \\
|u|_{\beta}^{2} \leq c\left(\lambda_{0}\right)|u|_{\alpha}^{2}, \quad \text { for all } u \in \mathcal{H}_{\alpha},
\end{gathered}
$$

where $c\left(\lambda_{0}\right)>0$ and $0<\lambda_{0}<\beta$.

\section{GLOBAL SOLUTIONS: EXISTENCE AND UNIQUENESS}

In order to obtain global existence and uniqueness for solution of the Cauchy problem (1.2) we will introduce the following hypotheses:

$$
(A u, u) \geq \beta|u|^{2}, \quad \text { for all } u \in D(A) \text { and } \beta>0,
$$

$$
\begin{gathered}
M \text { is locally lipschitz function on }\left[0,+\infty\left[\text { and } M(\xi) \geq m_{0}>0 \text {, for all } \xi \geq 0,\right.\right. \\
\int_{\boldsymbol{R}} e^{2 \lambda \eta}\left\|\mathcal{U}\left(u_{0}\right)\right\|_{\mathcal{H}(\lambda)}^{2} d \nu(\lambda)<+\infty \text { and } \int_{\boldsymbol{R}} e^{2 \lambda \eta}\left\|\mathcal{U}\left(u_{1}\right)\right\|_{\mathcal{H}(\lambda)}^{2} d \nu(\lambda)<+\infty
\end{gathered}
$$

for some $\eta>0$, where $e^{2 \lambda \eta}=\exp (2 \lambda \eta)$ and $\mathcal{U}$ is the unitary operator of (2.4) and (2.5).

We have from the hypothesis (3.1) that $\operatorname{supp}(\nu) \subset] \lambda_{0},+\infty\left[, 0<\lambda_{0}<\beta\right.$. Thus, let us define the space of the functions that satisfies (3.3) as follows

$$
W=\left\{w \in H ; \int_{\lambda_{0}}^{\infty} e^{2 \lambda \eta} \mid\|\mathcal{U}(w)\|^{2} d \nu(\lambda)<+\infty, \text { for some } \eta>0\right\}
$$

The space $W$ is not empty. In fact, the functions of the type $\mathcal{U}(w)=w_{\delta}=e^{-(\eta+\delta) \lambda}$, for $\delta>0$ or $w \cdot w_{\delta}$ with $w \in L^{1}(0, T ; \mathcal{H}(\lambda))$ belongs to $W$. In particular when the Hilbert space 
$H$ is the Euclidean space $\mathbb{R}$, the operator $\mathcal{U}$ is the Fourier transform operator $\mathcal{F}$ and $u(x)$ is the function $u(x)=\frac{1}{a^{2}+|x|^{2}}, a=\eta+\delta>0$, then $u$ belongs to $W$, because $\mathcal{F} u(x)=\sqrt{2 \pi} e^{-a|\lambda|}$. The convolution $w(x)=(u * v)(x)$ with $w \in L^{2}(\mathbb{R})$, are also example of functions of $W$. Similarly we obtain functions in $\mathbb{R}^{n}, n \geq 2$.

The vector space $V$ is identified with the Hilbert space:

$$
V=\left\{v \in D\left(A^{\frac{m}{2}}\right) ;\left|A^{\frac{m}{2}} v\right|^{2} \leq C \Lambda^{m} \cdot m !, \text { for some } \Lambda>0, c>0, m \in \mathbb{N}\right\}
$$

The characterization of space $W$ with the space $V$ is given by:

PROPOSITION 3.1: If $W$ and $V$ are spaces defined in (3.4) and (3.5) respectively, then $V \equiv W$.

PROOF. We have by (2.5) (Diagonalization Theorem), in particular, that

$$
\begin{gathered}
\mathcal{U}: D\left(A^{\frac{m}{2}}\right) \longleftrightarrow \mathcal{H}_{\frac{m}{2}} \text { is a isomorphism and } \\
|u|_{D\left(A \frac{m}{2}\right)}^{2}=|\mathcal{U}(u)|_{\frac{m}{2}}^{2}=\int_{\boldsymbol{R}} \lambda^{m}|| \mathcal{U}(u) \|^{2} d \nu(\lambda), \quad \forall u \in D\left(A^{\frac{m}{2}}\right) .
\end{gathered}
$$

Thus, if $u \in W$, then from the inequality $\frac{(2 \lambda \delta)^{m}}{m^{m}} \leq e^{2 \lambda \delta}$, we have

$$
\lambda^{m} \leq m !\left(\frac{1}{2 \delta}\right)^{m} \cdot e^{2 \lambda \delta}
$$

and

$$
\int_{\boldsymbol{R}} \lambda^{m}\|\mathcal{U}(u)\|^{2} d \nu(\lambda) \leq m !\left(\frac{1}{2 \delta}\right)^{m} \int_{\boldsymbol{R}} e^{2 \lambda \delta}\|\mathcal{U}(u)\|^{2} d \nu(\lambda)<+\infty .
$$

Therefore, $W \hookrightarrow V$.

Reciprocaly, if $u \in V$, we have

$$
\int_{\boldsymbol{R}} \lambda^{m} \mid \mathcal{U}(u) \|^{2} d \nu(\lambda) \leq C \Lambda^{m} m !
$$

or

$$
\int_{R}\left(\frac{\lambda}{2 \Lambda}\right)^{m} \cdot \frac{1}{m !}\|\mathcal{U}(u)\|^{2} d \nu(\lambda) \leq \frac{C}{2^{m}}
$$

Therefore,

$$
\int_{\boldsymbol{R}} e^{\frac{\lambda}{2 \lambda}}\|\mathcal{U}(u)\|^{2} d \nu(\lambda)<+\infty
$$

Thus $V \hookrightarrow W$.

With this caracterization the vectors of $W$ are of $A$-analytic type. 


\subsection{THE MAIN RESULT}

THEOREM 3.1: We fix $T>0$ an arbitrary real number. We have that if the hypothesis (3.1)-(3.3) are valid, then problem (1.2) has an unique global classical solution $u:[0, T[\rightarrow H$, satisfying

$$
u \in C^{2}([0, T] ; V),
$$

where $V$ is the Hilbert space defined in (3.5).

Suppose (3.1) then by Diagonalization theorem, there exists an unitary operator $\mathcal{U}$ from $H$ onto $\mathcal{H}_{0}$ such that $\mathcal{U}$ is an isomorphism from $D\left(A^{\alpha}\right)$ onto $\mathcal{H}_{\alpha}$, for $\alpha \in \mathbb{R}$, and

$$
\mathcal{U}\left(A^{\alpha} u\right)=\lambda^{\alpha} \mathcal{U}(u), \quad \text { for all } u \in D\left(A^{\alpha}\right)
$$

Moreover, $u$ is a solution of the problem (1.2) if and only if $v=\mathcal{U}(u)$ is solution of:

$$
\left\{\begin{array}{l}
v^{\prime \prime}+M\left(|v|_{\frac{1}{2}}^{2}\right) \lambda v=0, \quad t \geq 0 \\
v(0)=v_{0}=\mathcal{U}\left(u_{0}\right) ; \quad v^{\prime}(0)=v_{1}=\mathcal{U}\left(u_{1}\right) .
\end{array}\right.
$$

In such a problem, $v_{0}$ and $v_{1}$ belong to space $W$.

Now, our goal is to prove the existence and uniqueness of global solution $v$ for (3.7) in the following class

$$
v \in C^{2}\left([0, T] ; \mathcal{H}_{\alpha}\right)
$$

where $T>0$ and $\alpha \in \mathbb{R}$.

\subsection{TRUNCATED PROBLEM (LOCAL SOLUTION)}

Let $k \in \mathbb{N}$ and denote by $\mathcal{H}_{0, k}$ the subspace of $\mathcal{H}_{0}$ of the vector fields $v(\lambda)$ such that $v(\lambda)=0, \nu$-a.e. on $\left[k,+\infty\left[\right.\right.$. It follows that $\mathcal{H}_{0, k}$ equipped with the norm of $\mathcal{H}_{0}$ is a Hilbert space. For each vector field $v \in \mathcal{H}_{\alpha}, \alpha \in \mathbb{R}$, we denote by $v_{k}$ the truncated field associated to $v$, defined in the following way:

$$
v_{k}= \begin{cases}v, \nu-\text { a.e. } & \text { on }] \lambda_{0}, k\left[, 0<\lambda_{0}<\beta\right. \\ 0, \nu \text { a.e. } & \text { on }[k,+\infty[\end{cases}
$$

where $\beta>0$ is the constant of the (3.1).

It is not difficult to prove that $v_{k} \in \mathcal{H}_{0, k}$ and that $v_{k} \rightarrow v$ strongly in $\mathcal{H}_{\alpha}$. 
The truncated problem corresponding to (3.7)-(3.8) consists of finding $v_{k}:\left[0, T_{k}\right] \rightarrow \mathcal{H}_{0, k}$ such that

$$
v_{k} \in C^{2}\left([0, T] ; \mathcal{H}_{0, k}\right), \quad T>0
$$

satisfying

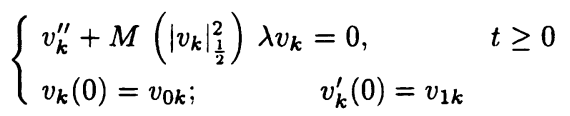

Let $V_{k}=\left[\begin{array}{l}v_{k} \\ v_{k}^{\prime}\end{array}\right]$, then (3.10) it is equivalent to:

$$
\left\{\begin{array}{l}
\frac{d}{d t} V_{k}=F\left(V_{k}\right), \quad t \geq 0 \\
V_{k}(0)=V_{0 k},
\end{array}\right.
$$

where

$$
F\left(V_{k}\right)=\left[\begin{array}{c}
v_{k}^{\prime} \\
-M\left(\left|v_{k}\right|_{\frac{1}{2}}^{2}\right) \lambda v_{k}
\end{array}\right] \quad \text { and } \quad V_{0 k}=\left[\begin{array}{c}
v_{0 k} \\
v_{1 k}
\end{array}\right]
$$

As $M$ is locally Lipschitz function, then $F^{\prime}$ is also locally Lipschitz.

Therefore by Cauchy-Lipschitz-Picard Theorem follows that there exists $0<T_{k}<T$ and an unique local solution $V_{k}$ of (3.11) in the class $C^{1}\left(\left[0, T_{k}\right] ; \mathcal{H}_{0, k} \times \mathcal{H}_{0, k}\right)$. Hence, there exist an unique $v_{k}:\left[0, T_{k}\right] \rightarrow \mathcal{H}_{0, k}$ solution of (3.10), satisfying

$$
v_{k} \in C^{2}\left(\left[0, T_{k}\right] ; \mathcal{H}_{0, k}\right)
$$

Our next objective will be to obtain estimates in order to extend the solutions $v_{k}$ to all interval $\left[0, T\left[, T>0\right.\right.$, and to prove that $v_{k}$ converge uniformly to $v$ solution of (3.7).

\section{Estimate "A Priori"}

Let us consider the linear equation

$$
v_{k}^{\prime \prime}+a_{k}(t) \lambda v_{k}=0, \quad t \geq 0
$$

where $a_{k}(t)$ is a real continuous and nonnegative function on $[0, T[$.

We will assume that all solutions of problem (3.10) also satisfy the linear equation (3.13).

Let us introduce the following function:

$$
E_{k}(t)=\frac{1}{2}\left\{\left\|v_{k}^{\prime}\right\|^{2}+\lambda\left\|v_{k}\right\|^{2}\right\}
$$

where $\|\cdot\|=\|\cdot\|_{\mathcal{H}(\lambda)}$. 
LEMMA 3.1: If $v_{k}$ is a solution of equation (3.13) on $\left[0, T\left[\right.\right.$, then $E_{k}$ satisfy:

$$
E_{k}(t) \leq c(T, \delta) E_{k}(0) \exp (\lambda \delta), \quad \text { for any } \quad \delta>0 .
$$

PROOF. Let us $\rho_{\varepsilon}(t)$ a smoothness net with $\left.\operatorname{supp}\left(\rho_{\varepsilon}\right) \subset\right] 0, \varepsilon\left[\right.$, then one defines $a_{k \varepsilon}(t)=$ $\left(\tilde{a}_{k} * \rho_{\varepsilon}\right)(t)+\sigma_{\varepsilon}$, where

$$
\begin{gathered}
\tilde{a}_{k}= \begin{cases}a_{k}, & \text { if } t \in[0, T] \\
0, & \text { if } t>T ;\end{cases} \\
\left(\tilde{a}_{k} * \rho_{\varepsilon}\right)(t)=\int_{\boldsymbol{R}} \tilde{a}_{k}(s) \rho_{\varepsilon}(t-s) d s \text { and } \sigma_{\varepsilon}=\varepsilon+\int_{0}^{t}\left|a_{k}-\tilde{a}_{k} * \rho_{\varepsilon}\right| d \xi, \quad k \in \mathbb{N}^{*} .
\end{gathered}
$$

In this conditions, we have

$$
a_{k \varepsilon} \in C^{1}([0, T]), a_{k \varepsilon}>0, a_{k \varepsilon} \rightarrow a_{k} \text { uniformly on }[0, T]
$$

and using the inequality

$$
\int_{0}^{T} \frac{\left|a_{k \varepsilon}-a_{k}\right|}{\sqrt{a_{k \varepsilon}}} d t \leq \int_{0}^{T} \frac{\left|a_{k \varepsilon}-\tilde{a}_{k} * \rho_{\varepsilon}\right|}{\sqrt{a_{k \varepsilon}}} d t+\int_{0}^{T} \frac{\sigma_{\varepsilon}}{\sqrt{a_{k \varepsilon}}} d t
$$

we have by Lebesgue's Dominated Convergence Theorem that:

$$
\int_{0}^{T} \frac{\left|a_{k \varepsilon}-a_{k}\right|}{\sqrt{a_{k \varepsilon}}} d t \rightarrow 0 \quad \text { when } \quad \varepsilon \rightarrow 0^{+} .
$$

Let us define the auxiliarity $\varepsilon$-functions:

$$
E_{k \varepsilon}(t)=\frac{1}{2}\left\{\left\|v_{k}^{\prime}\right\|^{2}+\lambda a_{k \varepsilon}\left\|v_{k}\right\|^{2}\right\}
$$

Differentiating $E_{k \varepsilon}$ and using (3.13) we obtain

$$
\frac{d}{d t} E_{k \varepsilon}=\frac{1}{2} \lambda a_{k \varepsilon}\left\|v_{k}\right\|^{2} \frac{a_{k \varepsilon}^{\prime}}{a_{k \varepsilon}}+\lambda\left(\left(v_{k}, v_{k}^{\prime}\right)\right)\left[a_{k \varepsilon}-a_{k}\right],
$$

hence, by using $E_{k \varepsilon}$ and standard inequality we have

$$
\frac{d}{d t} E_{k \varepsilon} \leq\left[\frac{\left|a_{k \varepsilon}^{\prime}\right|}{a_{k \varepsilon}}+\lambda \frac{\left|a_{k \varepsilon}-a_{k}\right|}{\sqrt{a_{k \varepsilon}}}\right] E_{k \varepsilon}
$$

By Gronwall's inequality, we conclude

$$
E_{k \varepsilon}(t) \leq E_{k \varepsilon}(0) \exp \left[\int_{0}^{T}\left(\frac{\left|a_{k \varepsilon}^{\prime}\right|}{a_{k \varepsilon}}+\lambda \frac{\left|a_{k \varepsilon}-a_{k}\right|}{\sqrt{a_{k \varepsilon}}}\right) d t\right] .
$$

As $a_{k \varepsilon}$ is inferiorly bounded by $\varepsilon$ on $[0, T]$, then we may compare $E_{k}$ with $E_{k \varepsilon}$, that is,

$$
c_{1}(\varepsilon) E_{k}(t) \leq E_{k \varepsilon}(t) \leq c_{2}(\varepsilon) E_{k}(t) . \quad\left(c_{1}(\varepsilon)>0\right) .
$$


Moreover,

$$
a_{k \varepsilon}^{\prime}=\left(\tilde{a}_{k} * \frac{d}{d t} \rho_{\varepsilon}\right)+\left|a_{k}-\tilde{a}_{k} * \rho_{\varepsilon}\right|
$$

is bounded on $[0, T]$, therefore

$$
\int_{0}^{T} \frac{\left|a_{k \varepsilon}^{\prime}\right|}{a_{k \varepsilon}} d t \leq C=C(T, \varepsilon)
$$

Hence, from (3.17) and (3.18) we infer that

$$
E_{k}(t) \leq c(T, \varepsilon) E_{k}(0) \exp \left(\lambda \int_{0}^{T} \frac{\left|a_{k \varepsilon}-a_{k}\right|}{\sqrt{a_{k \varepsilon}}} d t\right)
$$

Using (3.16) in (3.19) it follows:

$$
E_{k}(t) \leq c(T, \delta) E_{k}(0) \exp (\lambda \delta), \quad \text { for all } t \geq 0
$$

and $\delta>0$. As required.

The next step is to obtain a estimate of the $v_{k}$ in $\mathcal{H}_{\frac{1}{2}}$.

Taking scalar product of both sides in (3.10) with $v_{k}^{\prime}$ we have

$$
\left(v_{k}^{\prime \prime}, v_{k}^{\prime}\right)_{0}+M\left(\left|v_{k}\right|_{\frac{1}{2}}^{2}\right)\left(\lambda v_{k}, v_{k}^{\prime}\right)_{0}=0, \text { for all } 0 \leq t \leq T_{k}
$$

Taking $\hat{M}(\sigma)=\int_{0}^{\sigma} M(s) d s$, we obtain

$$
\frac{1}{2} \frac{d}{d t} E_{k}(t)=0
$$

where

$$
E_{k}(t)=\frac{1}{2}\left\{\left|v_{k}^{\prime}\right|_{i}^{2}+\hat{M}\left(\left|v_{k}\right|_{\frac{1}{2}}^{2}\right)\right\}
$$

Integrating from 0 to $t \leq T_{k}$ it follows

$$
E_{k}(t)=E_{k}(0)
$$

But, by hypothesis $(3.2), \hat{M}\left(\left|v_{k}\right|_{\frac{1}{2}}^{2}\right) \geq m_{0}\left|v_{k}\right|_{\frac{1}{2}}^{2}$, thus

$$
\left|v_{k}^{\prime}(t)\right|_{0}^{2}+m_{0}\left|v_{k}(t)\right|_{\frac{1}{2}}^{2} \leq\left|v_{1 k}\right|_{0}^{2}+\hat{M}\left(\left|v_{o k}\right|_{\frac{1}{2}}^{2}\right)=\int_{\lambda_{0}}^{\infty}\left\|v_{1 k}\right\|^{2} d \nu(\lambda)+\int_{0}^{\left|v_{0 k}\right|_{\frac{1}{2}}^{2}} M(s) d s
$$

On the other hand, from (3.3) it follows

$$
\int_{\lambda_{0}}^{\infty}\left\|v_{1 k}\right\|^{2} d \nu(\lambda) \leq \int_{\lambda_{0}}^{\infty} e^{2 \lambda \eta}\left\|v_{1 k}\right\|^{2} d \nu(\lambda)<+\infty
$$


and

$$
\left|v_{0 k}\right|_{\frac{1}{2}}^{2}=\int_{\lambda_{0}}^{\infty} \lambda\left\|v_{0 k}\right\|^{2} d \nu(\lambda) \leq \int_{\lambda_{0}}^{\infty} e^{2 \lambda \eta}\left\|v_{0 k}\right\|^{2} d \nu(\lambda)<+\infty
$$

As $M$ is locally lipschitz, $\int_{0}^{\left|v_{0 k}\right|_{\frac{1}{2}}^{2}} M(s) d s<+\infty$. Hence, we conclude from (3.20) that

$$
\left|v_{k}^{\prime}(t)\right|_{0}^{2}+\left|v_{k}(t)\right|_{\frac{1}{2}}^{2} \leq c, \text { for all } t \geq 0
$$

where $c$ is a positive constant that depends only on the initial data.

Multiplying (3.15) by $\lambda \geq \lambda_{0}>0$ and integrating in relation the measure $\nu$ on $] \lambda_{0},+\infty[$, by using the hypothesis (3.3) we obtain

$$
\begin{aligned}
\left|v_{k}^{\prime}\right|_{\frac{1}{2}}^{2}+\left|v_{k}\right|_{1}^{2} & \leq c(T, \delta)\left\{\int_{\lambda_{0}}^{\infty} e^{2 \lambda \delta}\left\|v_{1 k}\right\|^{2} d \nu(\lambda)+\int_{\lambda_{0}}^{\infty} e^{2 \lambda \delta}\left\|v_{0 k}\right\|^{2} d \nu(\lambda)\right\} \\
& \leq c, \quad \text { for all } t \in[0, T[.
\end{aligned}
$$

REMARK 3.1: From (3.21) we conclude that the solution $v_{k}$ of (3.10) can be extended to the whole $\left[0, T\left[\right.\right.$, for any $T>0$. Therefore, it follows from (3.12) that $v_{k}$ satisfies (3.9).

\section{Limit of the Truncated Solutions}

In order to take the limit in (3.10) when $k \rightarrow \infty$ it is necessary to prove the following result.

LEMMA 3.2: The sequence of the functions $\left(v_{k}\right)_{k \in N}$ is the Cauchy in $\mathcal{H}_{\alpha}, \alpha \in \mathbb{R}$.

PROOF. If $v_{k}$ and $v_{\jmath}$, for $k>j$ are solutions of the (3.13), then $w_{k}=v_{k}-v_{\jmath}$ satisfy

$$
w_{k}^{\prime \prime}+a_{k} \lambda w_{k}=\left(a_{\jmath}-a_{k}\right) \lambda v_{k} .
$$

Associated to the equation (3.23), let us denote by $F_{k}(t)$ the function given by

$$
F_{k}(t)=\frac{1}{2}\left\{\left\|w_{k}^{\prime}\right\|_{\mathcal{H}(\lambda)}^{2}+\lambda\left\|w_{k}\right\|_{\mathcal{H}(\lambda)}^{2}\right\}
$$

It is immediate that,

$$
F_{k}(0)=0, \quad \text { for all } k \in \mathbb{N}
$$

Our next goal is to prove that $F_{k}(t) \rightarrow 0$ when $k \rightarrow \infty$. In fact, as in the proof of Lemma 3.1, let $\rho_{\varepsilon}(t)$ a smoothness sequence with $\left.\operatorname{supp}\left(\rho_{\varepsilon}\right) \subset\right] 0, \varepsilon\left[\right.$ and $a_{k \varepsilon}(t)=\left(\tilde{a}_{k} * \rho_{\varepsilon}\right)(t)+\sigma_{k \varepsilon}$, where

$$
\tilde{a}_{k}= \begin{cases}a_{k}, & \text { if } \quad t \in[0, T] \\ 0, & \text { if } \quad t>T\end{cases}
$$




$$
\left(\widetilde{a}_{k} * \rho_{\varepsilon}\right)(t)=\int_{\boldsymbol{R}} \tilde{a}_{k}(s) \rho_{\varepsilon}(t-s) d s \quad \text { and } \quad \sigma_{k \varepsilon}=\varepsilon+\int_{0}^{T}\left|a_{k}-\widetilde{a}_{k} * \rho_{\varepsilon}\right| d t
$$

In this conditions (e.g. (3.10)), we have

$$
\int_{0}^{T} \frac{\left|a_{k \varepsilon}-a_{\varepsilon}\right|}{\sqrt{a_{k \varepsilon}}} d t \rightarrow 0 \quad \text { when } \quad \varepsilon \rightarrow 0^{+}, \text {for fix } k
$$

Associated to the $a_{k \varepsilon}$ we define the function:

$$
F_{k \varepsilon}(t)=\frac{1}{2}\left\{\left\|w_{k}^{\prime}\right\|_{\mathcal{H}(\lambda)}^{2}+\lambda a_{k \varepsilon}\left\|w_{k}\right\|_{\mathcal{H}(\lambda)}^{2}\right\}
$$

Deriveting $F$ with respect to time, we obtain

$$
F_{k \varepsilon}^{\prime}=\frac{1}{2} \lambda a_{k \varepsilon}^{\prime}\left\|w_{k}\right\|^{2}+\lambda a_{k \varepsilon}\left(\left(w_{k}, w_{k}^{\prime}\right)\right)+\left(\left(w_{k}^{\prime \prime}, w_{k}^{\prime}\right)\right)
$$

Using the equation (3.23), we have:

$$
\begin{aligned}
F_{k \varepsilon}^{\prime} & =\frac{1}{2} \lambda a_{k \varepsilon}^{\prime}\left\|w_{k}\right\|^{2}+\lambda\left[a_{k \varepsilon}-a_{k}\right]\left(\left(w_{k}, w_{k}{ }^{\prime}\right)\right)+\left(\left(\left(a_{\jmath}-a_{k}\right) \lambda v_{\jmath}, w_{k}{ }^{\prime}\right)\right) \\
& \leq \frac{1}{2} \lambda a_{k \varepsilon}\left\|w_{k}\right\|^{2} \frac{\left|a_{k \varepsilon}^{\prime}\right|}{a_{k \varepsilon}}+\lambda^{\frac{1}{2}} \frac{\left|a_{k \varepsilon}-a_{k}\right|}{\sqrt{a_{k \varepsilon}}} \lambda^{\frac{1}{2}} \sqrt{a_{k \varepsilon}}\left\|w_{k}\right\|\left\|w_{k}{ }^{\prime}\right\| \\
& +\left|a_{\jmath}-a_{k}\right| \lambda\left\|\mid v_{j}\right\|\left\|w_{k}^{\prime}\right\| \\
& \leq \frac{1}{2} \lambda a_{k \varepsilon}\left\|w_{k}\right\|^{2} \frac{\left|a_{k \varepsilon}^{\prime}\right|}{a_{k \varepsilon}}+\lambda^{\frac{1}{2}} \frac{\left|a_{k \varepsilon}-a_{k}\right|}{\sqrt{a_{k \varepsilon}}} \frac{1}{2}\left[\lambda a_{k \varepsilon}\left\|w_{k}\right\|^{2}+\left\|w_{k}{ }^{\prime}\right\|^{2}\right] \\
& +\frac{1}{2}\left|a_{\jmath}-a_{k}\right|^{2} \lambda^{2}\left\|v_{\jmath}\right\|^{2}+\frac{1}{2}\left\|w_{k}^{\prime}\right\|^{2} .
\end{aligned}
$$

It follows that

$$
F_{k \varepsilon}^{\prime} \leq\left[\frac{\left|a_{k \varepsilon}^{\prime}\right|}{a_{k \varepsilon}}+\lambda^{\frac{1}{2}} \frac{\left|a_{k \varepsilon}^{\prime}-a_{k}\right|}{\sqrt{a_{k \varepsilon}}}+1\right] F_{k \varepsilon}+\frac{1}{2}\left|a_{j}-a_{k}\right|^{2} \lambda^{2}\left\|v_{j}\right\|^{2} .
$$

From (3.15) we have that $\left\|v_{j}\right\|^{2} \leq c$, for all $j \in \mathbb{N}$. Thus, integrating from 0 to $t \leq T$ and applying Gronwall inequality, we get in the similar way as Lemma 3.1 that

$$
F_{k \varepsilon}(t) \leq c(T, \delta) F_{k \varepsilon}(0) \exp (\lambda \delta)+c(T, \delta) \exp (\lambda \delta)\left|a_{k}-a_{\jmath}\right|_{L^{2}(0, T)}^{2}
$$

Using (3.18) and (3.24) it follows that

$$
F_{k}(t) \leq c(T, \delta) \exp (\lambda \delta)\left|a_{k}-a_{j}\right|_{L^{2}(0, T)}^{2}
$$

By hypothesis (3.3) and for $\delta>0$, small enough, we have

$$
e^{\delta \lambda} F_{k}(t) \leq c(T, \delta) e^{2 \lambda \delta}\left|a_{k}-a_{j}\right|_{L^{2}(0, T)}^{2}
$$

where $e^{\lambda \delta}=\exp (\lambda \delta)$. 
From (3.21) and (3.22) it follows

$$
v_{k} \in C^{0}\left([0, T] ; \mathcal{H}_{\frac{1}{2}}\right)
$$

Thus, the sequence of function $\phi_{k}(t)=\left|v_{k}(t)\right|_{\frac{1}{2}}^{2}$ is continuous in $[0, T]$. On the other hand, given $s, t \in[0, T]$, we get

$$
\left|\phi_{k}(t)-\phi_{k}(s)\right|_{R}^{2} \leq c\left|v_{k}(t)-v_{k}(s)\right|_{\frac{1}{2}}^{2} \leq c \int_{s}^{t}\left|v_{k}^{\prime}(\eta)\right|_{\frac{1}{2}}^{2} d \eta \leq c|t-s|^{2}
$$

for all $k$ and $0 \leq t, s \leq T$.

Hence, by Arzelá-Áscoli Theorem there exists a subsequence $\left(\phi_{k}\right)_{k \in \boldsymbol{N}}$, which we yet denote by $\left(\phi_{k}\right)_{k \in N}, \phi \in C^{0}([0, T] ; \mathbb{R})$ such that

$$
\phi_{k} \rightarrow \phi, \text { uniformely in } C^{0}([0, T] ; \mathbb{R}) .
$$

As $M$ is locally lipschitz it follows that

$$
M\left(\left|v_{k}\right|_{\frac{1}{2}}^{2}\right) \rightarrow M(\phi) \text { in } C^{0}([0, T] ; \mathbb{R})
$$

Taking $k, j \rightarrow \infty$ and remarking that $a_{k}(t)=M\left(\left|v_{k}\right|_{\frac{1}{2}}^{2}\right)$ it follows

$$
\left|a_{k}(t)-a_{j}(t)\right|_{L^{2}(0 . T)}^{2} \rightarrow 0
$$

Thus,

$$
e^{\lambda \delta} F_{k}(t) \rightarrow 0, \quad \text { for all } t \geq 0 .
$$

Therefore, $\left(v_{k}\right)_{k \in N}$ is a Cauchy sequence in $\mathcal{H}_{\alpha}, \alpha \in \mathbb{R}$.

Integrating (3.26) in relation to the measure $\nu$ on $] \lambda_{0},+\infty$ [, we obtain

$$
v_{k} \rightarrow v \quad \text { strongly in } \quad C^{0}\left([0, T] ; \mathcal{H}_{\alpha}\right),
$$

for $\alpha \in \mathbb{R}$.

As $M$ is locally Lipschitz, follows from (3.27) that

$$
M\left(\left|v_{k}\right|_{\frac{1}{2}}^{2}\right) \rightarrow M\left(|v|_{\frac{1}{2}}^{2}\right) \quad \text { em } \quad C^{0}([0, T] ; \mathbb{R}) .
$$

Using (3.27) and (3.28) in (3.10), we have

$$
v_{k}^{\prime \prime} \rightarrow v^{\prime \prime} \quad \text { strongly in } \quad C^{0}\left([0, T] ; \mathcal{H}_{\alpha}\right)
$$

Therefore, from (3.27)-(3.29) we conclude that $v=\mathcal{U}(u)$ satisfies (3.7) and (3.8). 


\section{PROOF OF THEOREM 3.1}

The operator $\mathcal{U}: D\left(A^{\alpha}\right) \rightarrow \mathcal{H}_{\alpha}, \alpha \in \mathbb{R}$, is an isomorphism. Thus, from (3.7) and (3.8) we have that the vector function $u:\left[0, T\left[\rightarrow H\right.\right.$ defined by $u=\mathcal{U}^{-1}(v)$ satisfies the problem (1.2) and

$$
u \in C^{2}\left([0, T] ; D\left(A^{\alpha}\right)\right)
$$

Now, let us verify that the vector field $u(t)$ belongs to Hilbert space $V$ defined in (3.5). Thus, we affirm that the application

$$
t \longmapsto\|u(t)\|_{V}^{2} \in C^{2}([0, T])
$$

In fact, from Proposition 3.1 is sufficient to prove that $u \in W$, is that,

$$
\int_{\lambda_{0}}^{\infty} e^{\lambda \eta}\|\mathcal{U}(u)\|^{2} d \nu(\lambda)<+\infty
$$

The function $E(t)$ is given by

$$
E(t)=\frac{1}{2}\left\{\left\|v^{\prime}\right\|^{2}+\lambda\|v\|^{2}\right\}=\frac{1}{2}\left\{\left\|\mathcal{U}(u)^{\prime}\right\|^{2}+\lambda\|\mathcal{U}(u)\|^{2}\right\},
$$

and from Lemma 3.1, follows

$$
\begin{aligned}
\int_{\lambda_{0}}^{\infty} e^{\lambda \delta}\|\mathcal{U}(u)\|^{2} d \nu(\lambda) & \leq \int_{\lambda_{0}}^{\infty} e^{\lambda \delta} E(t) d \nu(\lambda) \\
& \leq c(T, \eta) \int_{\lambda_{0}}^{\infty} e^{2 \lambda \delta}\left\{\left\|\mathcal{U}\left(u_{1}\right)\right\|^{2}+\left\|\mathcal{U}\left(u_{0}\right)\right\|^{2}\right\} d \nu(\lambda) \leq \\
& \leq c=c\left(\mathcal{U}\left(u_{1}\right) ; \mathcal{U}\left(u_{0}\right)\right) .
\end{aligned}
$$

Therefore, $u \in C^{2}([0, T] ; V)$.

In order to accomplish the Proof of Theorem 3.1, let us prove that the solution of Problem (1.2) is unique. Or equivalently, that the solution of Problem (3.7) is unique. In fact, if $v_{1}$ and $v_{2}$ are two solutions of problem (3.7), then $w=v_{1}-v_{2}$ satisfy

$$
\left\{\begin{array}{l}
w^{\prime \prime}+M\left(\left|v_{1}\right|_{\frac{1}{2}}^{2}\right) \lambda w=\left[M\left(\left|v_{2}\right|_{\frac{1}{2}}^{2}\right)-M\left(\left|v_{1}\right|_{\frac{1}{2}}^{2}\right)\right] \lambda v_{1} \text { in } C^{0}([0, T] ; V) \\
w(0)=w^{\prime}(0)=0
\end{array}\right.
$$

From (3.31) let us define a function

$$
G_{\varepsilon}(t)=\frac{1}{2}\left\{\left\|w^{\prime}(t)\right\|^{2}+a_{\varepsilon}(t) \lambda\|w(t)\|^{2}\right\}
$$


where $a_{\varepsilon}(t)=\left(M\left(\left|v_{1}\right|_{\frac{1}{2}}^{2}\right) * \rho_{\varepsilon}\right)(t)+\varepsilon, \varepsilon>0$.

Deriveting $G_{\varepsilon}(t)$, we obtain

$$
G_{\varepsilon}^{\prime}(t)=\left(\left(w^{\prime}, w^{\prime \prime}\right)\right)+a_{\varepsilon}^{\prime}(t) \lambda\|w\|^{2}+a_{\varepsilon}(t) \lambda\left(\left(w^{\prime}, w\right)\right),
$$

using the equation $(3.31)_{1}$, we get

$$
\begin{aligned}
G_{\varepsilon}^{\prime}(t) & =\lambda\left[a_{\varepsilon}(t)-M\left(\left|v_{1}\right|_{\frac{1}{2}}^{2}\right)\right]\left(\left(w^{\prime}, w\right)\right)+\left[M\left(\left|v_{1}\right|_{\frac{1}{2}}^{2}\right)-M\left(\left|v_{2}\right|_{\frac{1}{2}}^{2}\right)\right] \\
& \cdot\left(\left(\lambda v_{1}, w^{\prime}\right)\right)+a_{\varepsilon}^{\prime}(t) \lambda\|w\|^{2} .
\end{aligned}
$$

As $M$ is locally Lipschitz, follows

$$
\left|M\left(\left|v_{1}\right|_{\frac{1}{2}}^{2}\right)-M\left(\left|v_{2}\right|_{\frac{1}{2}}^{2}\right)\right| \leq c(T)|w|_{\frac{1}{2}}, \quad \text { for all } t \in[0, T] .
$$

By the substitution of (3.34) in (3.33) and using (3.32), we obtain

$$
G_{\varepsilon}^{\prime}(t) \leq\left[\frac{a_{\varepsilon}(t)-M\left(\left|v_{1}\right|_{\frac{1}{2}}^{2}\right)}{\sqrt{a_{\varepsilon}(t)}}\right] G_{\varepsilon}(t)+\frac{\left|a_{\varepsilon}^{\prime}(t)\right|}{a_{\varepsilon}(t)} G_{\varepsilon}(t)+c \lambda\left\|v_{1}\right\|\left\|w^{\prime}\right\||w|_{\frac{1}{2}} .
$$

Taking

$$
r_{\varepsilon}(t)=\left[\frac{a_{\varepsilon}(t)-M\left(\left|v_{1}\right|_{\frac{1}{2}}^{2}\right)}{\sqrt{a_{\varepsilon}(t)}}\right]+\frac{\left|a_{\varepsilon}^{\prime}(t)\right|}{a_{\varepsilon}(t)}
$$

and multiplying (3.35) for $e^{-\int_{0}^{t} r_{\epsilon}(s) d s}$, we have

$$
\frac{d}{d t}\left\{G_{\varepsilon}(t) e^{-\int_{0}^{t} r_{\varepsilon}(s) d s}\right\} \leq\left. c \lambda\left\|v_{1}\right\|\left\|w^{\prime}\right\||| w\right|_{\frac{1}{2}} e^{-\int_{0}^{t} r_{\varepsilon}(s) d s} .
$$

Integrating from 0 to $t$,

$$
G_{\varepsilon}(t) e^{-\int_{0}^{t} r_{\varepsilon}(s) d s} \leq c \lambda \int_{0}^{t}\left\|v_{1}\right\|\left\|w^{\prime}\right\||w|_{\frac{1}{2}} e^{-\int_{0}^{t} r_{\varepsilon}(s) d s} d \xi,
$$

or, still

$$
G_{\varepsilon}(t) \leq c \lambda \int_{0}^{t}\left\|v_{1}\right\|\left\|w^{\prime}\right\||w|_{\frac{1}{2}} d \xi
$$

Integrating in relation to the measure $\nu$ on $] \lambda_{0},+\infty[$ and denoting by $J(t)=$ $\int_{\lambda_{0}}^{\infty} G_{\varepsilon}(t) d \nu(\lambda)$, we obtain

$$
J(t) \leq c \int_{0}^{t}\left[|w|_{\frac{1}{2}} \cdot \int_{\lambda_{0}}^{\infty} \lambda\left\|v_{1}\right\|\left\|w^{\prime}\right\| d \nu(\lambda)\right] d \xi
$$

Using the Hölder inequality and the estimate (3.21), it follows that

$$
\int_{\lambda_{0}}^{\infty} \lambda\left\|v_{1}\right\|\left\|w^{\prime}\right\| d \nu(\lambda) \leq c\left(\int_{\lambda_{0}}^{\infty}\left\|w^{\prime}\right\|^{2} d \nu(\lambda)\right)^{\frac{1}{2}} \leq c \sqrt{J(t)} .
$$


Substituting in (3.36) we conclude that

$$
J(t) \leq c \int_{0}^{t}|w|_{\frac{1}{2}} \sqrt{J(\xi)} d \xi \leq \tilde{c} \int_{0}^{t} J(\xi) d \xi
$$

Using the Gronwall's inequality, we get

$$
J(t)=0, \quad \text { for all; } t \geq 0 .
$$

Therefore,

$$
w(t)=0, \quad \text { for all } t \geq 0 .
$$

REMARK 3.2: Without the hypothesis (2.3), i.e., considering only $(A u, u) \geq 0, \forall u \in D(A)$, our main result (Theorem 3.1) can be proved. In this case, we take the operator $A_{\varepsilon}=A+\varepsilon I$, where $I$ represents the identity operator and $\varepsilon>0$, which satisfies the conditions of the Diagonalization Theorem. The solution to (1.2) would be obtain as a limit of $u_{\varepsilon}$ solution to

$$
\begin{cases}u_{\varepsilon}^{\prime \prime}+M\left(\left|A_{\varepsilon}^{\frac{1}{2}} u_{\varepsilon}\right|^{2}\right) & A_{\varepsilon} u_{\varepsilon}=0 \\ u_{\varepsilon}(0)=u_{0}, & u_{\varepsilon}^{\prime}(0)=u_{1}\end{cases}
$$

If the operator $A=-\Delta$ in $\mathbb{R}^{n}$ we have to consider $A_{\varepsilon}=-\Delta+\varepsilon I$ to apply the Diagonalization Theorem.

Acknowledgement- The author is very grateful to the referee for his valuable suggestions which improved this paper.

\section{REFERENCES}

[1] AROSIO, A; SPAGNOLO, S. - "Global solution of the Cauchy problem for a nonlinear hyperbolic equation", Nonlinear Partial Differential Equation and their Applications, Collège de France Seminar, Vol. 6 (ed. by H. Brezis and J.L. Lions), Pitman, London, (1984).

[2] CARRIER, G.F. - "On the vibration problem of elastic string", Q. J. Appl. Math. 3, pp. 151-165 (1945).

[3] DICKEY, R.W. - "Infinite systems of nonlinear oscillations equations related to string", Proc. A.M.S. 23, pp. 459-469 (1969).

[4] EBIHARA, Y.; MEDEIROS, L.A. \& MILLA MIRANDA, M. - "Local solutions for a nonlinear degenerated hyperbolic equations", Nonlinear Analysis 10, pp. 27-40 (1986). 
[5] HUET, D. - Décomposition spectrale et opérateurs, Presses Universitaires de France (1977).

[6] LIONS, J.L. \& MAGENES, E. - Problémes aux limıtes non homogènes et applıcatıons, Vol. 1, Dunod, Paris (1968).

[7] LIONS, J.L. - Quelques méthodes de résolutıon des problèmes aux lımıtes non linéaires, Dunod, Paris (1969).

[8] LIONS, J.L. - On some questions in boundary value problems of mathematrcal physics, in Contemporary Development in Continuous Mechanics and Partial Differential Equations (ed. by G. de la Penha, L.A. Medeiros), North Holland, London (1978).

[9] MATOS, P.M. - "Mathematical Analysis of the Nonlinear Model for the Vibrations of a String", Nonlinear Analysıs, Vol. 17, No. 12, pp.1125-1137 (1991).

[10] MEDEIROS, L.A. \& MILLA MIRANDA, M. - "Solution for the equation of nonlinear vibration in Sobolev space of fractionary order", Mat. Aplic. Comp., Vol. 6, № 3, pp. 257-276 (1987).

[11] MENZALA, G.P. - "On classical solution of a quasilinear hyperbolic equation", Nonlinear Analysıs, Vol. 3, № 5, pp. 613-627 (1978).

[12] NISHIHARA, K. - "Degenerate quasilinear hyperbolic equation with strong damping", Funcialaj Ekvacioj 27, pp. 125-145 (1984).

[13] POHOZAEV, S. - "On a class of quasilinear hyperbolic equations", Math. Sbornik 95, pp. 152-166 (1975).

[14] YAMADA, Y. - "Some nonlinear degenerate wave equations", Nonlınear Analysıs, Vol. 11, № 10, pp. 1155-1168 (1987). 


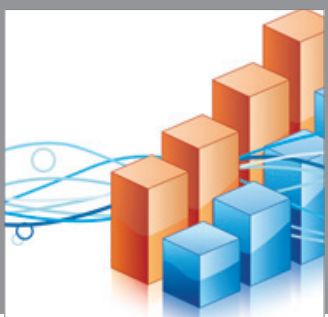

Advances in

Operations Research

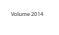

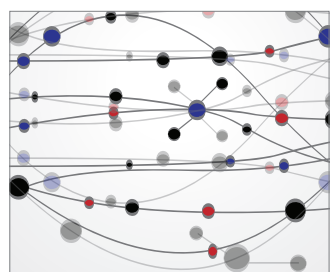

\section{The Scientific} World Journal
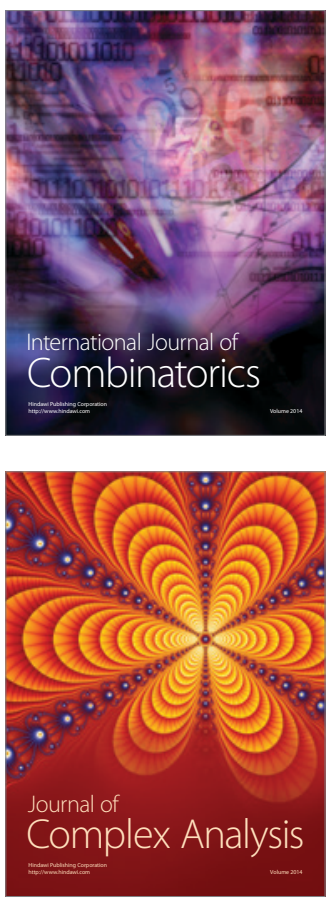

International Journal of

Mathematics and

Mathematical

Sciences
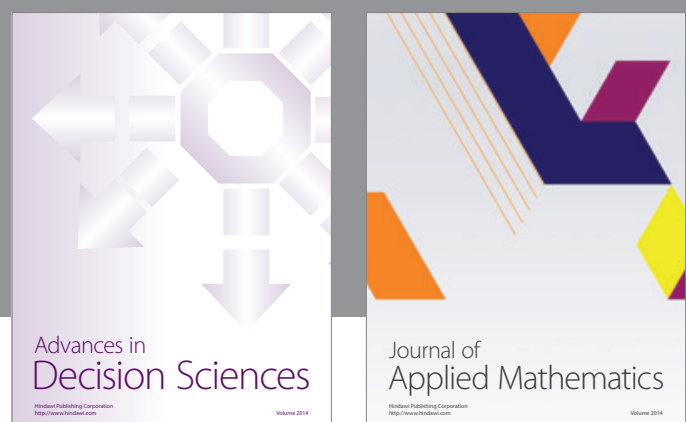

Journal of

Applied Mathematics
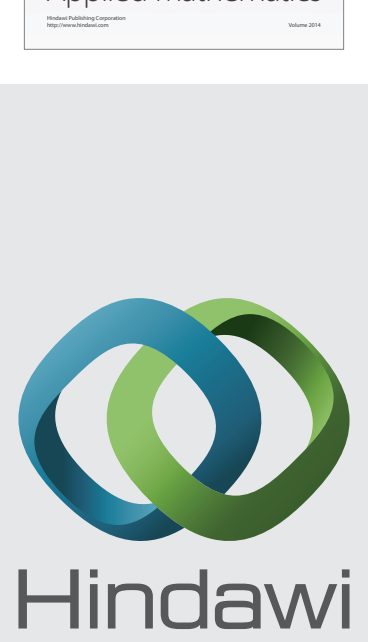

Submit your manuscripts at http://www.hindawi.com
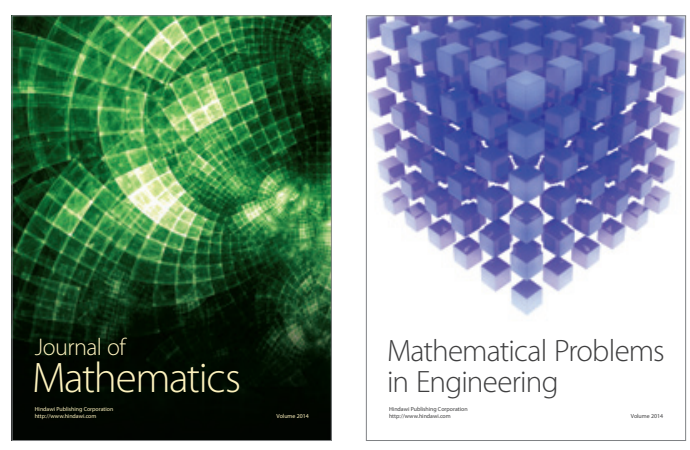

Mathematical Problems in Engineering
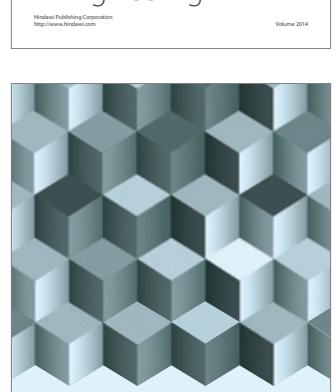

Journal of

Function Spaces
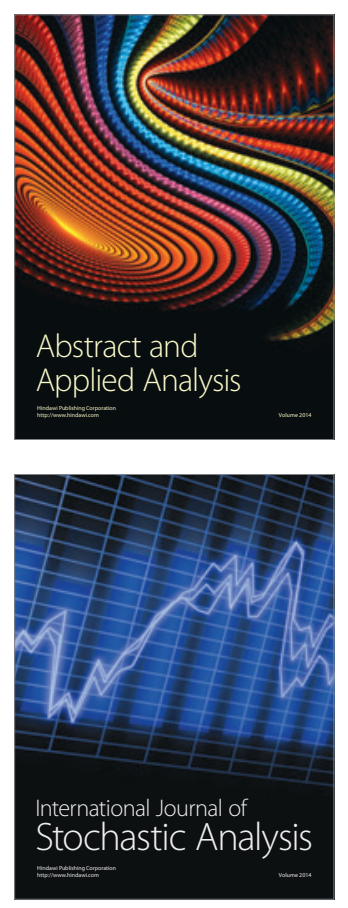

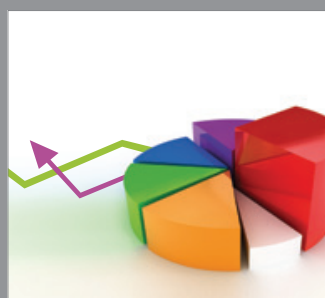

ournal of

Probability and Statistics

Promensencen
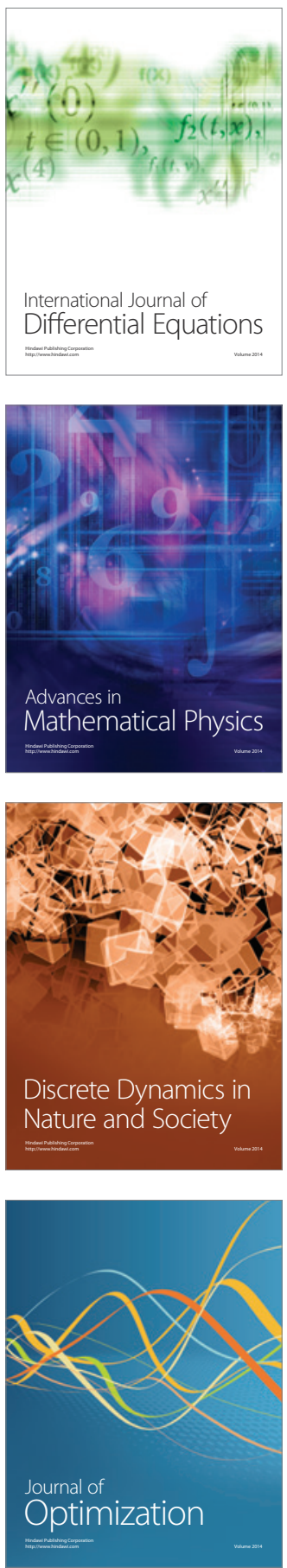